\title{
Testing Hypotheses About Glacial Dynamics Using a Statistical Model of Paleo-Climate
}

Robert Kaufmann ( $\square$ kaufmann@bu.edu )

Boston University https://orcid.org/0000-0002-5670-7027

\section{Felix Pretis}

University of Victoria

\section{Research Article}

Keywords: Glacial cycles, linear, nonlinearity, termination, Mid-Brunhes event

Posted Date: April 27th, 2021

DOl: https://doi.org/10.21203/rs.3.rs-459880/v1

License: (9) This work is licensed under a Creative Commons Attribution 4.0 International License. Read Full License 
2 Testing Hypotheses About Glacial Dynamics Using a Statistical Model of Paleo-Climate

3 Enter authors here: Robert K. Kaufmann¹, Felix Pretis ${ }^{2}$

$4{ }^{1}$ Department of Earth and Environment, Boston University, Boston, Massachusetts, USA, 02215

5 OCRID ResearcherID:I-4962-2017

6

$7 \quad 2$ Department of Economics, University of Victoria, Victoria, BC, Canada; and Nuffield College,

8 University of Oxford, Oxford, UK ORCID 0000-0003-1435-9295

9

10 Corresponding author: Robert K. Kaufmann (Kaufmann@bu.edu)

11 Key Words: Glacial cycles, linear, nonlinearity, termination, Mid-Brunhes event 


\section{Abstract}

15 We test hypotheses about glacial dynamics by evaluating the ability of a linear statistical model to 16 simulate climate during the previous $\sim 800,000$ years. During this period, the linear model 17 simulates the timing and magnitude of glacial cycles, including the saw-tooth pattern in which ice 18 accumulates gradually and ablates rapidly, without falsely simulating an interglacial after each

19 peak in obliquity. Conversely, the linear model fails to simulate experimental observations that are 20 created by a nonlinear data generating process. Together, these (in)abilities suggest that 21 nonlinearities, threshold effects, bifurcations, and/or phase-specific governing equations do not 22 play a critical role in glacial cycles during the late Pleistocene. Furthermore, the model's accuracy 23 throughout the sample period suggests that changes in orbital geometry create the Mid-Brunhes 24 event. 


\section{Introduction}

When considered over the last eight-hundred thousand years, climate shows highly persistent patterns. Most notable are glacial cycles. During glaciations, temperature, greenhouse gas concentrations, and sea level remain below their sample mean for extended periods; during these same periods, land and sea ice remain above their sample means (Kaufmann and Juselius, 2013). These positions are reversed for extended periods known as interglacials. These persistent movements and complex climate dynamics create difficulties for statistical analyses of climate data. Using ordinary least squares to analyze time series that show persistent movements tends to greatly inflate findings of a statistically meaningful relations among time series when none are present (Yule, 1929; Engle and Granger, 1987).

Difficulties posed by highly persistent movements and complex dynamics are alleviated using vector-autoregression, cointegration, and equilibrium correction (Kaufmann and Juselius, 2013). Using these methods, Kaufmann and Juselius (2013), herein KJ2013, estimate a linear statistical model of climate from a sample that includes observations from the previous 391 thousand years. The model, termed a cointegration vector autoregression (CVAR), specifies linear relations between four exogenous variables for orbital geometry; eccentricity $(E c c)$, obliquity $(O b l)$,

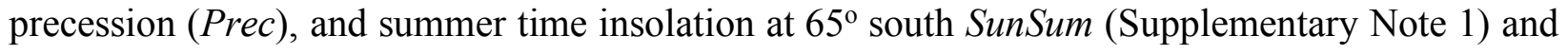
ten endogenous climate variables; Antarctic land (Temp) and sea surface temperature (SST), carbon dioxide (CO2) and methane ( $\mathrm{CH} 4)$ concentrations, land (Ice) and sea ice ( $\mathrm{Na}$ ), sea level (Level), iron dust $(\mathrm{Fe})$, and non sea-salt sulfate $(\mathrm{SO} 4)$ and calcium $(\mathrm{Ca})$, which are chosen to proxy physical relations thought to drive glacial cycles (Kaufmann and Juselius, 2013, Supplementary Note II). The CVAR model explicitly represents linear long-run equilibrium relations between orbital geometry and climate, which are given by ten cointegrating relations, and climate dynamics, which are given by constant rates at which the climate system adjusts towards the equilibrium given by the long-run (cointegrating) relations. A similar approach is applied to a subset of climate variables (Davidson et al., 2016).

As described in Kaufmann and Juselius $(2013 ; 2016)$ as well as Kaufmann and Pretis (2021), these statistical relations validate some basic hypotheses about the mechanisms that are postulated to drive glacial cycles (e.g. carbon dioxide affects temperature via radiative forcing), reproduce 
the main features of glacial cycles (e.g. the timing, magnitude, and saw-tooth pattern of changes in land ice volume), and separate observed interglacial periods from skipped/missing beats (e.g. Huybers, 2012), which are peaks in insolation (e.g. obliquity) that do not generate deglaciations and thus interglacials. Over the last million years, twelve of the twenty-five peaks in obliquity are not associated with deglaciations (Tzedakis et al., 2017).

To date, efforts to explain missing beats in particular, and glacial cycles in general, focus on nonlinear relations (e.g. Tziperman et al., 2006), threshold effects (e.g. Paillard, 1998; 2001, Ganopolski et al., 2016), bifurcations (e.g. Ashwin and Ditlevsen, 2015) and/or phase-specific governing equations (e.g. Tzedakis et al., 2017). Some of these dynamics are embodied in several models that account for many features of the glacial cycle (e.g. Parrenin and Paillard, 2012; Paillard and Parennin, 2004). Based on these successes, there is a consensus that nonlinearities, threshold effects, bifurcations, and/or phase specific governing equations play a critical role in glacial cycles in general and terminations in particular, as summarized by a recent review "Terminations clearly represent a strongly nonlinear response to regional changes in the seasonality of solar radiation (Past interglacials Working Group of Pages, 2016).”

Here, we explore this consensus using a linear CVAR model reported by KJ2013. Specifically, is the success of previous models based on their inclusion of nonlinearities, threshold effects, bifurcations, or phase-specific governing equations, etc? To test this hypothesis, we evaluate whether a model that omits nonlinearities, threshold effects, bifurcations, and/or phase-specific governing equations can accurately simulate glacial cycles. To answer, we test whether a statistical model that specifies linear relations among orbital geometry and climate variables can simulate glacial cycles accurately. If a linear model can simulate glacial cycles accurately, the logic of Occam's razor implies that nonlinearities, threshold effects, bifurcations, and/or phase-specific governing equations are complexities that do not play a critical in glacial cycles beyond linear relations. Conversely these complexities likely play an important role if a simple linear model without nonlinearities, threshold effects, bifurcations, and/or phase-specific governing equations cannot accurately simulate glacial cycles. Specifically, we formulate and test three hypotheses.

1. To establish the capabilities of the CVAR model, we asses the degree to which the linear specification of the CVAR model is able to simulate non-linear relations among experimental data that are simulated by non-linear Van der Pol Oscillators. We postulate that a linear CVAR model will not be able to simulate non-linear relations among the 
experimental data. Consistent with this hypothesis, the linear CVAR model is not able to simulate the non-linear relations among an exogenous forcing, which mimics orbital geometry, and two endogenous variables which mimic components of climate that react quickly (temperature) and slowly (ice volume) to changes in orbital geometry.

2. We assess the ability of the linear CVAR to simulate (out-of-sample and in-sample) ten endogenous variables that represent important components of the observed paleo-climate record based on four variables that represent orbital geometry. We postulate that the linear CVAR model will fail to simulate glacial cycles accurately if nonlinearities, threshold effects, bifurcations, and/or phase-specific governing equations play an important role in the timing and magnitude of glacial cycles. But if their role is small relative to linear relations, we postulate that the linear CVAR model will simulate glacial cycles accurately, both during the in-sample period used to estimate the model (391 Kyr BP - present) and an out-of-sample period (792 Kyr BP - $392 \mathrm{Kyr} \mathrm{BP}$ ). Compared to its poor performance with the nonlinear experimental data generated by Van der Pol oscillators, the linear CVAR model is able to account for a much larger portion of the variation in climate variables during both the in- and out-of-sample periods. Furthermore, the model errors are distributed randomly between interglacials and the other phases of the glacial cycle. This performance suggests that nonlinearities, threshold effects, bifurcations, or changes in governing equations do not play a critical role in the timing or magnitude of glacial cycles.

3. We assess the ability of the linear CVAR model to simulate glacial cycles before and after the Mid-Brunhes event (MBE), in which $\mathrm{CO}_{2}$ concentrations rise, the amplitude interglacials increase, and interglacials are cooler but longer starting about 430 thousand years before the present (EPICA, et al., 2004; Luthi et al., 2008; Hoenisch et al., 2009, Jansen et al., 1986). If changes in orbital geometry play an important role in the MBE, there will be little change in the ability of the linear CVAR model to simulate glacial cycles before and after the MBE because the CVAR model specifies the same relations between orbital geometry and climate before and after the MBE. But if changes in long- and short run relations between orbital geometry and climate variables create the changes in glacial cycles that are associated with the MBE, the CVAR's ability to simulate glacial cycles before the MBE will be diminished relative to the period after the MBE. This is because the linear CVAR is estimated from observations after the MBE and uses that these long- 
and short-run relations to simulate the period before the MBE. Results indicate that model performance does not change around the MBE, which suggests that the MBE is driven by changes in orbital geometry.

These results and the methods used to obtain them are described in five sections. Section 2 describes the data and methods used to generate and analyze the simulations. The results are reported in section 3 , discussed in section 4 , and section 5 concludes.

\section{Methods}

\subsection{The CVAR Model Specifies Linear Relations Among Variables}

The equations used by the linear CVAR model to estimate long- and short-run relations between orbital geometry and climate are given by:

$$
\Delta x_{t}=A_{0} \Delta w_{t}+A_{1} \Delta w_{t-1}+\Gamma_{1} \Delta x_{t-1}+\Gamma_{2} \Delta x_{t-2}+\Pi z_{t-1}^{\prime}+\varepsilon_{t}
$$

in which $x_{t}$ is a $10 \times 1$ vector that includes the ten endogenous variables; Temp, CO2, CH4, Ice, $\mathrm{Fe}, \mathrm{Na}, \mathrm{Ca}$, SO, Level, and SST (Table 1); $w$ is a $4 \times 1$ vector that includes the four exogenous variables for orbtial geometry Ecc, Prec, Obl, and Sunsum, $z^{\prime}=\left[x_{t}^{\prime}, w_{t}^{\prime}, 1\right], \Gamma_{1}(10 \times$ $10), A_{0}(10 \times 4), A_{1}(10 \times 4)$, are matrices of short-run coefficients; $\Pi$ is a $10 \times 15$ matrix of long-run coefficients, $\Delta$ is the first difference operator $\left(\Delta x_{t}=x_{t}-x_{t-1}\right)$, and $\varepsilon_{i}$ is an error term with mean value zero and variance $\Omega$ that is normally, independently, and identicially distributed.

The condition that the conditional process $\left(x_{t} \mid w_{t}\right)$ is nonstationary is formulated as a reduced rank hypothesis on the matrix П:

$$
\Pi=\alpha \beta^{\prime}
$$

in which $\alpha$ is a $10 \times r$ matrix of coefficients, which describe a constant rate at which the ten climate variables (or $I$ and $T$ in the experimental data set) adjust towards the equilibrium that is given by orbital geomtery (or $F$ in the experimental data set); $r$ is the number of cointegration relations given by the reduced rank of the $\Pi$ matrix and $\beta$ is a $r \times 15$ matrix of cointegration coefficients that define the $r$ stationary deviations from long-run equilibrium relations, the so called cointegration relations, $\beta^{\prime} z_{t}$. Using maximum likelihood techniques, KJ2013 estimate the $\beta$ and $\alpha$ matrices (Supplementary Tables 2 and 3) KJ2013 from a partial system (Johansen 1992, Harbo et al., 1998, Juselius 2006) in which orbital variables are weakly exogenous (i.e. changes in climate do not affect orbital geometry). To identify the equations and generate standard errors for the statistical parameters, KJ2013 impose 26 overidentifying restrictions on the $\beta$ matrix. No 
restrictions are imposed on the CVAR model that is estimated from the experimental data to maximize its ability to simulate $I$ and/or $T$ (hypothesis 1 ).

Despite its complexity, the CVAR model is linear in parameters. Equilibrium relations, as represented by the cointegrating relations $\beta^{\prime} z_{t}$ specify linear relations (in levels) among variables, and changes in variables are modelled as linear functions of disequilibrium. To illustrate, the tenth cointegrating relation in KJ2013 (Supplementary Table 2) represents a linear long-run equilibrium relation between Ice and orbital geometry which can be used to represent the equilibrium level of Ice that is implied by orbital geometry as follows:

$$
I_{c} e_{\mathrm{t}}=-0.755^{*} E c c_{t}+4.459 * O \text { Obl }_{\mathrm{t}}+2.881 * \text { SunSum }_{\mathrm{t}}
$$

Statistically significant coefficients associated with $\mathrm{Obl}$ and SunSum indicate that obliquity has information about Ice that is not contained in SunSum and vice-versa, despite their strong correlation. Similarly, there are strong correlations among the four variables for orbital geometry, but statistically significant coefficients indicate that no combination of three contains all of the information in the fourth.

System dynamics are represented by adjustment towards equilibrium $(\alpha)$, which is constant (over all phases of the glacial cycle) and is a linear function of disequilibrium in the level of the variables in the previous time period. For Ice, 3.7 percent of the disequilibrium between the previous period's equilibrium value (as implied by the tenth cointegrating relation equation (3)) and the previous period's value is eliminated each period:

$$
\Delta \text { Ice }_{t}=-0.037 \times\left(\text { Ice }_{t-1}-\left(-0.755 * E c c_{t-1}+4.459 * E c c_{t-1}+2.881 * \text { SunSum }_{t-1}\right)\right)
$$

A constant rate of adjustment 3.7 percent implies a constant adjustment time, which does not represent the nonlinearities of ice flow and mass balance for an ice sheet (Roe, 2006). Roe (2006) argues that these nonlinearities are critical "the nonlinearities of ice flow and mass balance preclude the application of a single adjustment time scale to an ice sheet." This contention is test by hypothesis 2 . If nonlinearities associated with ice sheet and other aspects of the climate are important, their omission will prevent the linear CVAR model from simulating ice volume accurately. Conversely if the nonlinearities of ice flow and mass balance for an ice sheet do not play a critical role in glacial cycles, the linear CVAR model may accurately simulate ice volume in particular, and glacial cycles in general.

Equations 1 and 2 specify many parameters, but the statistical model is not 'overfit.' In KJ2013, each of the ten dependent variables in $x_{t}$ has 390 observations. Each equation has 357 degrees of 
179 freedom because the $\Pi, \Gamma, A_{0}$ and $A_{1}$ matrices specify 33 coefficients for each of the ten equations.

180 These 33 coefficients correspond to the 15 columns in the $\Pi$ matrix (including a constant), the 10 181 columns in the $\Gamma$ matrix, and the four columns in the $A_{o}$ and $A_{1}$ matrices. Of these 33 coefficients, 182 many are not statistically different from zero (Supplemental Table 4). As a result, the ten equations contain between 7 and 16 variables per equation, which is consistent with the range suggested by Maasch and Saltzman (1990). Thus, the $\sim 357$ degrees of freedom alleviates any concern the the equations are 'overfit.'

Finally, the correlation among the ten endogenous climate variables has little effect on the model's ability to simulate glacial cycles. The model is driven by the four exogenous variables for orbital geometry; the model has no information about any of the climate variables beyond the values used to initialize the model. Under these conditions, the statistical relations in the linear CVAR model translate changes in orbital geometry into changes in climate but do not contain information about climate beyond that contained in the variables for orbital geometry.

To make all data amenable to a statistical analysis, KJ2013 converts observations from the proxy record to a common time scale (EDC3) using conversions from Parrenin et al., (2007) and Ruddiman and Raymo (2003). Unevenly spaced observations are interpolated (linearly) to generate a data set in which each series has a time step of $1 \mathrm{kyr}$, which has relatively little effect on results (Miller, 2019). To eliminate the effects of inverting matrices with elements that differ greatly in size (due to different units of measurement), each of the fourteen time series is standardized as follows:

$x_{i}=\left(y_{t}-\bar{y}\right) / \sqrt{\operatorname{Var}(y),} \quad t=1, \ldots, 391$

in which $y_{t}$ is the value (in original units), $\bar{y}$ is the mean value over the $391 \mathrm{Kyr}$ in-sample period, and $\operatorname{Var}(y)$ is the variance over the in-sample period. Equation (5) also is used to normalize the 1000 observations in the experimental data generated by the Van der Pol oscillators (Section 2.2). We recognize that nonstationary time series do not have a constant mean or variance, rather the sample mean and variances are used in a linear transformation to harmonize the values of the time series.

\subsection{Generating Experimental Data with Non-Linear Relations}

To test whether the linear CVAR model can simulate non-linear processes, we use the CVAR methodology to estimate the relation among experimental data that are generated by non-linear van der Pol oscillators (Crucifix, 2013). Using the parameters specified by Crucifix (2013), van- 
210 der-Pol oscillators create experimental data that mimic paleo-climate data. Specifically, a

211 sinusoidal forcing $F$, which mimics changes in orbital geometry, is perturbed with white noise to

212 simulate a variable ' $T$ ' that responds rapidly to $F$ (such as temperature), while variable ' $I$ ' mimics

213 a variable that responds gradually (such as ice). This process is used to create 1,000 observations

214 in discrete time for each variable (Figure 1(a) \& (b)). We use the first 500 observations to estimate

215 the CVAR model. The remaining 500 observations constitute an out-of-sample period.

216

217

218

219

220

221

222

223

224

225

226

227

228

229

230

231

232

233

234

235

236

237

238

239

240

\subsection{Simulating Experimental Data or Observed Climate Variables using a Linear CVAR Model}

To assess hypothesis 1-3, we simulate the linear CVAR model of climate over a $\sim 800 \mathrm{kyr}$ sample period, which includes a $391 \mathrm{Kyr}$ BP - present in-sample period that used to estimate the model (Kaufmann and Juselius, 2013) and a 792 Kyr BP - 392 Kyr BP out-of-sample period. To simulate climate during the in- and out-of-sample periods, the ten endogenous variables $x$ (or two experimental data simulated by the Van der Pol Oscillators) are expressed as a function of the exogenous variables for orbital geometry (or the exogenous variable used to drive the Van der Pol Osciallators) and shocks to the climate system $(\varepsilon)$ by inverting Equation (1) into the moving average form:

$x_{t}=C \sum_{i=1}^{t} \varepsilon_{t}+C^{*}(L) \varepsilon_{t}+C_{w} w_{t}+C_{w}^{*}(L) \Delta w_{t}$

In which $C=\beta_{\perp}\left(1-\Gamma_{1}\right)^{-1} \alpha_{\perp} ; \alpha_{\perp}$ is a $10 \times(10-r)$ matrix orthogonal to $\alpha$ describing the stochastic trends and $\beta_{\perp}$ is a $10 \times(10-r)$ matrix orthogonal to $\beta$ determining how the stochastic trends load into the climate variables; $L$ is the lag operator (for example, $\left.L \varepsilon_{t}=\varepsilon_{t-1}\right) ; C^{*}(L)$ and $C_{w}^{*}(L)$ are stationary lag polynomials; $C_{w}$ is $10 \times 4$; and the matrices are functions of the parameters $\left(A_{0}, A_{1}, \Gamma_{1}, \alpha, \beta\right)$. Based on the ten cointergating relations reported by $\mathrm{KJ} 2013 r=10$, then $C=0$, the in- and out-of-sample simulations are based on model (2) subject to (3) by setting $\varepsilon_{t}=0$ which implies that the simulated variables, $\hat{x}_{t}$, are calculated from the exogenous drivers, $C_{w} w_{t},\left(A_{0} \Delta w_{t}\right)$, the dynamics attached to them, $C_{w}^{*}(L) \Delta w_{t-1},\left(A_{1} \Delta w_{t-1}\right)$, and the internal climate dynamics $C^{*}(L) \varepsilon_{t}\left(\Gamma_{1} \Delta \hat{x}_{t-1}, \alpha \beta^{\prime z_{t-1}}\right)$.

The out-of-sample simulation is initialized using observed values for Temp, SST, and Ice, which are available starting $800 \mathrm{kyr} \mathrm{BP}$. The time series for $\mathrm{CO}_{2} \mathrm{CH}_{4}, \mathrm{Fe}, \mathrm{Na}, \mathrm{SO} 4, \mathrm{Ca}$, and Level have more recent start dates (Table 1). For these variables, the model is initialized with values that correspond to their sample mean. We use these values to 'spin up' the model so that the simulated values converge towards observed values and looses sensitvity to initial conditions. Following this $10 \mathrm{Kyr}$ 'spin-up,' which is shorter than other climate models (e.g. Parrenin and Paillard, 2012), the 
model is run continuously through the present. Because the simulation starts the out-of-sample period, this ordering adds rigor. That is, error in the out-of-sample simulation are passed to the start of the in-sample simulation.

\subsection{Assessing model performance}

There are many ways to assess model performance. Previous analyses use spectral analyses, which evaluate the degree to which the power spectrum of the observed and simulated data match (e.g. Ditlevsen et al., 2020). These efforts focus on the $100 \mathrm{Kyr}$ frequency. But this emphasis is made difficult by the paucity of observations; by definition, there are only eight possible peaks at the $100 \mathrm{Kyr}$ frquency in the $800 \mathrm{Kyr}$ record that are recorded in cores recovered from the Antarctic ice.

Instead, we focus on the model's ability to simulate each of the 792 observations for each of the 1 Kyr time steps. First, we quantify the model's skill in replicating glacial cycles based on the size of errors as measured by root mean square error (RMSE) $\sqrt{\frac{\sum_{i=1}^{T}\left(x_{t}-\hat{x}_{t}\right)^{2}}{T}}$ in which $\hat{x}_{t}$ is the value for variable $x$ at time t simulated by the model, $x_{t}$ is the observed value for the proxy, and $\mathrm{T}$ is the number of observations. The fraction of variation that is explained by the linear CVAR model is quantified by the adjusted $\mathrm{r}^{2}$, which is estimated from the following regression:

$$
x_{t}=\kappa+\varphi \hat{x}_{t}+\mu_{t}
$$

in which $\kappa$ and $\varphi$ are regression coefficient estimated using ordinary least squares and $\mu$ is the regression residual. For ice, the $\mathrm{r}^{2}$ quantifies the fraction of variation that is observed in the 792 observations that is explained by the 792 values for Ice that are simulated by the linear CVAR model.

For each variable $x$, we use the simulation errors $\left(v_{t}=x_{t}-\hat{x}_{t}\right)$ to identify periods when model accuracy changes in a statistically significant fashion, such as stage $11^{1}$. We identify these periods with an indicator saturation technique [R-package gets Pretis et al., 2018; Castle et al., 2015]. This approach is used to assess the time-varying performance of climate models (Pretis et al., 2015), the forecast accuracy of economic predictions (Ericsson 2017), and the presence of volcanic eruptions in temperature reconstructions in both simulated climate data (Pretis et al., 2016) and proxy-reconstructions (Schneider et al., 2017).

\footnotetext{
${ }^{1}$ Stage 11 is a well-known mismatch between orbital geometry and an interglacial period (e.g. Imbrie et al., (1993)) that occurs between 424,000 and 374,000 years ago.
} 
This indicator saturation technique can identify the date(s) when the value for each of the ten variables simulated by the linear CVAR model $\hat{x}$ deviates significantly from $x$ (i.e. simulation errors are statistically different from zero) for a single time-step (outlier). Persisting errors are values of $\hat{x}$ that deviate significantly from $\mathrm{x}$ for two or more consecutive time-steps (Supplementary Note III). Outliers and persisting errors are evaluated for every possible time step. We retain only those outliers or persisting errors that exceed a $p \alpha=0.001$ threshold. This threshold implies that random chance will cause the technique to identify one outlier (or persisting error) per 1,000 observations. This tightly controls the false-positive rate of detected periods of model failure.

If model performance does not change in a systematic fashion through phases of the glacial cycle or over time, we expect outliers and persisting errors to occur randomly throughout the sample. For each of the ten endogenous variables (and as a group), we compare the distribution of outliers and persisting errors between interglacial and non-interglacial periods, as defined by Tzedakis et al., (2012) and between the in-sample and out-of-sample periods. We test whether the timing of outliers and persisting errors across the sample period is different from a uniform random distribution (expected under the null-hypothesis of equal performance) using a Pearson chi-square test $(\mathrm{P})$, which is calculated as follows:

$P=\sum_{j=1}^{n} \frac{\left(o_{j}-E_{j}\right)^{2}}{E_{j}}$

in which $n$ is two periods (interglacial $j=1$; non-interglacial $j=2$; or in-sample $j=1$; out-ofsample $j=2), O_{j}$ is the number of outliers or persisting errors that are identified in period $j$, and $E_{j}$ is the number of outliers or persisting errors that are expected in period $j$.

The number of outliers or persisting errors expected in period $j\left(E_{j}\right)$ is calculated based on the null hypothesis that outliers or persisting errors are distributed uniformly between periods. This null implies that the expected number outliers or persisting errors $\left(E_{j}\right)$ can be calculated as:

$E_{j}=\frac{Y r_{j}}{\sum_{i}^{n} Y r_{j i}} \times \sum_{j=1}^{n} O_{j}$

in which $Y r$ is the number of thousand-year time steps in period $j$ for which simulated and observed values are available and $n$ is the number of thousand year time steps in the simulation period. $P$ is evaluated against a $\chi^{2}$ distribution with n-1 degrees of freedom. If the test rejects the null hypothesis that outliers or persisting errors are distributed randomly between interglacial and noninterglacial phases of the cycle (i.e. some phases are simulated more/less accurately than others), 
the more accurate phase is identified by the numerator of Equation (7) $\left(O_{j}-E_{j}\right)$. A negative value for interglacial periods $\left(\left(O_{1}-E_{1}\right)<0\right)$ would indicate that the number of outliers or persisting errors detected during the interglacial phase of glacial cycles is less than expected by a uniform random distribution and hence the interglacial phase of glacial cycles is simulated more accurately than other phases of the glacial cycle.

\subsection{Testing Hypothesis 3: The Mid-Brunhes event (MBE) represents a change in the dynamics} that drive glacial cycles

The start of the in-sample period used to estimate KJ2013 falls close to the Mid-Brunhes event, which occurs about 430 thousand years before present (Jansen et al., 1986). To test hypothesis 3 , we compare the model performance before and after the MBE. Specifically, we compare the adjust $\mathrm{r}^{2}$, RMSE, and the distribution of outliers and persisting errors between the in- and out-of-sample periods. If these metrics indicate that the model performs equally well, this would be inconsistent with the hypothesis that the MBE represents a change in the long- and/or short-run relations in the climate system because these relations are held constant by the climate model. Instead, results that indicate model performance does not change would be consistent with the hypothesis that changes in orbital geometry drive the changes in glacial cycles that are associated with the MBE.

\section{Results \& Discussion}

\subsection{The linear CVAR model is not able to simulate non-linear relations}

Using the linear CVAR model to estimate the non-linear relations among $F, I$, and $T$ in the experimental data generates coefficients that are statistically different from zero (Supplementary Tables 5 and 6). But visual inspection of Figure 1(a-b) indicates that the resultant CVAR model is not able to simulate $T$ or $I$ during the in- or out-of-sample periods in a statistically meaningful fashion. Specifically, the simulated values for $T$ and $I$ account for a small portion of the variation in the $T$ and $I$ as measured by the $\mathrm{r}^{2}$ for equation (6) during any of the sample periods (Table 2). This poor performance implies that imposing a linear specification on the long- and short-run relations between the exogenous forcing $(F)$ and the endogenous variables $(I$ or $T)$ cannot capture the non-linear relation among these variables that is created by the nonlinear data generating process. This inability is not offset by the lagged short-run effects represented by coefficients in the A or $\Gamma$ matrices, which is set to two lags, which is consistent with the lag length used in KJ2013. Together, these failures indicate that the linear CVAR model cannot capture non-linear relations. 
The inability of the linear CVAR model to capture nonlinear relations among the experimental data (Figure 1(a-b)) suggests that the linear CVAR model can be used to test whether nonlinear processes, threshold effects, bifurcations, or changes in governing equations play an important role in glacial cycles. If nonlinear processes, threshold effects, bifurcations, or changes in governing equations play an important role in glacial cycles, the inability of the CVAR model to quantify nonlinear relations will prevent the CVAR from simulating glacial cycles accurately, as indicated by Figure 1 and the values for $\mathrm{r}^{2}$ in Table 2. Conversely, if nonlinear processes, threshold effects, bifurcations, and/or governing equations that vary by phase of the glacial cycle play a lesser role, and linear relations are largely responsible for the timing and magnitude of glacial cycles, a properly specified linear model, such as a CVAR, will be able to simulate glacial cycles more accurately than indicated in Figure 1 and Table 2.

\subsection{Hypothesis 1(b) Nonlinearities, threshold effects, and/or phase-specific governing equations} play an important role in the timing and magnitude of glacial cycles

For both the in- and out-of-sample periods, Figure 2 suggests that KJ2013 generally captures the timing and magnitude of persistent changes in climate that are described by glacial cycles. These cycles often are summarized by changes in land ice volume. Simulated values for Ice follow the saw-tooth pattern in which ice volume builds slowly but ablates rapidly (Figure 2d). Furthermore, the CVAR model generally simulates the timing and magnitude of changes in ice volume accurately (other than stage 11) without falsely simulating an integlacial after each peak in obliquity (i.e. missing beats). Consistent with these abilities, the $r^{2}$ values in Table 2 indicate that the linear CVAR model is able to account for about 60 percent of the variation in Ice and Temp dring the in-sample and out-of-sample periods (when stage 11 is excluded). This is considerably larger than the $\sim 20$ percent of the in-sample variation in temperature that is captured by a statistical model by Wunsch (2004). Similarly, the linear CVAR model is able to account for 20 - 60 percent of the variation in the eight other variables for climate, both in- and out-of-sample (Table 2). All of these values are larger than the portion of variation $(0.04-0.16)$ in the nonlinear experimental data that is simulated by the linear CVAR.

The visual suggestion in Figure 2 that the CVAR model is able to simulate all phases of glacial cycles with a modicum of accuracy is confirmed by tests that fail to reject the null hypothesis that outliers and persisting errors for Ice (and the other nine endogenous variables) are distributed randomly between interglacials and all other phases of glacial cycles (Table 3 ). This result suggests 
that nonlinearties, threshold effects, bifurcations, and/or changes in governing equations are not needed to simulate various phases of the glacial cycle.

The ability of the linear CVAR model to simulate seemingly non-linear changes in ice volume and the other nine variables likely stems from two sources. When a variable is far from equilibrium, the constant rate of adjustment $(\alpha)$ in the linear model moves the variable towards equilibrium by a larger amount than when that variable is closer to equilibrium. Second, the model is conditioned on orbital geometry, which changes nonlinearly over time. But the VAR model specifies these nonlinear changes with a linear relation between orbital geometry and climate variables. As such, non-linear changes in orbital geometry have a linear relation with the ten endogenous variables for climate that are simulated by the linear CVAR model.

The ability of the linear CVAR model to simulate all phases of the glacial cycles and the inand out-of-sample periods (see below) is inconsistent with the hypothesis that nonlinearties, threshold effects, bifurcations, and/or phase specific governing equations play a critical role in glacial cycles. Instead, results suggest that non-linear relations, thresholds, bifurcations, and/or changes in governing equations do not play a critical role in glacial cycles since the mid Pleistocene transition (MPT). Nonetheless, our results do not reject the presence of non-linear relations, thresholds, bifurcations, or changes in governing equations. We do not argue that linear relations govern ice flow and the mass balance; these processes clearly have a nonlinear component (e.g. Roe and Lindzen, 2001). But the linear CVAR model does not need to represent the nonlinear components of ice flow and mass balance to simulate glacial cycles accurately. Instead, the timing and magnitude of glacial cycles can be described by linear long- and short-run relations between orbital geometry and the climate system. Interpreting these results through the filter of Occams razor suggests that the current emphasis non-linear relations, thresholds, bifurcations, or changes in governing equations is misplaced; important drivers of glacial cycles can be understood using linear models.

Furthermore, the ability of the CVAR model to simulate climate during the out-of-sample period is inconsistent one of the extreme explanations that is listed by Tziperman et al., (2006) "glacial cycles would exist even in the absence of the insolation changes." If glacial cycles exist independently of changes in orbital geometry, a statistical model that is conditioned only on orbital geometry and spun up with no memory of previous cycles would not be able to simulate glacial cycles accurately during the initial out-of-sample period. Furthermore, if orbital geometry is held 
constant, the ten endogenous variables in the CVAR model come to an equilibrium and do not change thereafter, which is consistent with physically-based models (e.g. Ganopolski and Brovkin, 2017)

Finally, the model's ability to simulate glacial cycles during the out-of-sample period is inconsistent with speculation that the CVAR model's ability to reproduce the ten endogenous variables during the in-sample period simply reflects the model's ability to reproduce the data used to estimate the coefficients. Instead, the ability of the model to simulate climate during the out-ofsample period suggests that the regression coefficients in the $\Pi, \Gamma, A_{0}$ and $A_{1}$ matrices capture longand short-run relations among orbital geometry and the ten endogenous variables that govern the climate system for the $400 \mathrm{kyr}$ before the sample period used to estimate the model.

3.3 Testing hypothesis 3: The Mid-Brunhes event (MBE) represents a change in the dynamics that drive glacial cycles

The linear CVAR model is able to simulate glacial cycles during the in- and out-of-sample periods as indicated by root mean square error and $r^{2}$ (Table 2). As expected, the RMSE for the out-of-sample period generally is larger than the RMSE for the in-sample period. Consistent with this expectation, the $\mathrm{r}^{2}$ is larger for the in-sample period. But much of these differences are associated with MIS 11 (424-375 Kyr BP), most of which occurs during the out-of-sample period (Table 2). If we eliminate MIS 11 from the out-of-sample period, the RMSE and $\mathrm{r}^{2}$ of the in- and out-of-sample periods are similar (Table 2).

The small change in accuracy between the in- and out-of-sample periods is confirmed by the distribution of outliers and persisting errors (Figure 3). Tests indicate that we cannot reject the null hypothesis that outliers are distributed randomly between the in- and out-of-sample periods (Table 3). A test statistic $\chi^{2}(1)=0.09$ fails to reject $(p>0.76)$ the null hypothesis that as a group, outliers for the ten endogenous variables are distributed randomly between the in- and out-of-sample periods. Conversely, a test statistic $\chi^{2}(1)=52.5$ rejects $(\mathrm{p}<0.001)$ the null hypothesis that as a group, persisting errors for the ten endogenous climate variables are distributed randomly between the in- and out-of-sample periods. But this rejection may be somewhat misleading. The out-ofsample simulation for $\mathrm{CH} 4$ and Ice is more accurate than the in-sample simulation.

The relatively small changes in model performance for the in- and out-of-sample periods suggests that the MBE does not change the ability of the linear CVAR model to simulate glacial cycles. Compared to the in-sample period used to estimate KJ2013, the pre-MBE out-of-sample 
period has; (1) lower concentrations of $\mathrm{CO}_{2}$, (2) glacial cycles with a smaller amplitude, and (3) cooler but longer interglacial periods (EPICA, et al., 2004; Luthi et al., 2008; Hoenisch et al., 2009). These three changes can be caused by one or both of two possible mechanisms. Glacial cycles may change at the MBE due to changes in the orbital geometry that drive glacial cycles, "through a set of internal mechanisms, insolation alone induces a systematic difference between the interglacials before and after the $430 \mathrm{kyr}$ ago (Yin, 2013)." Alternatively, glacial cycles may change at the MBE due to changes in the endogenous dynamics that link orbital geometry to the climate system, "astronomical forcing alone cannot explain the difference in interglacial intensity before and after the MBE (Tzedakis et al., 2009).”

The CVAR model uses the linear long- and short-run relation estimated from the previous 391 kyr sample period to simulate the climate system before the MBE. The lack of a meaningful change in accuracy suggests that the same physical relations generate glacial cycles before and after the MBE. As such, our results are inconsistent with the hypothesis that "astronomical forcing alone cannot explain the difference in interglacial intensity before and after the MBE (Tzedakis et al., 2009)." Instead, the lack of a meaningful change in accuracy suggests that orbital geometry alone can account for the three changes in glacial cycles before and after the MBE.

\section{Conclusion}

Using only linear relations between orbital geometry and ten endogenous variables, the linear CVAR model is able to accurately simulate the evolution of climate during $\sim 400 \mathrm{kyr}$ in- and outof-sample periods during all phases of the glacial cycles. This ability suggests that non-linearities, thresholds, bifurcations, and/or changes in governing equations do not play a critical role in glacial cycles. Furthermore, there is little evidence that the MBE changes the underlying relations between orbital geometry and the climate system.

Nonetheless, the accuracy of the CVAR model declines in a statistically significant manner during MIS stage 11 and Termination V, which is consistent with arguments that the 'stage 11 paradox' is a mismatch between orbital geometry and climate (Imbrie et al., 1993). To investigate the causes for the 'stage 11 problem' we will analyze the statistical orderings of simulation errors to identify the equations that initiate the model's poor performance. In other words, the statistical ordering of simulation errors may allow the model to identify what is unique about MIS 11 (and termination V). 
The accuracy of the simulation also declines during the most recent portion of the Holocene, as indicated by persisting errors for Ice and SST (Figure 2(d) and 2(h)). Their errors indicate that the CVAR model understates the recent warming. This bias is consistent with the hypothesis that Holocene warming is amplified by anthropogenic emissions of carbon dioxide and methane (Ruddiman 2003; 2005; 2007) because these emissions are not included in the CVAR model. This omission suggests that we can use the CVAR model to test the Ruddiman hypothesis by quantifying the emissions of carbon dioxide and methane that eliminate the persisting errors for Ice and SST and comparing these emissions to independent estimates for early anthropogenic emissions (e.g. Stephens et al., 2019; Goldewijk et al., 2017).

Finally, the $\sim 800$ thousand year sample period includes observations after the Mid-Pleistocene transition (MPT). Future efforts will investigate the causes for the MPT by re-estimating the CVAR model with the proxy for ice volume compiled by Elderfield et al., (2012), simulating the model over the previous 1.57 million years, and comparing results with the method used to analyze the MBE. Following this logic, if the model fails to simulate ice volume before the MPT, the MPT represents a change in climate dynamics. Conversely, if the model is able to simulate ice volume before the MPT accurately, this would suggest that glacial cycles are generated by the same longand short-run relations before and after the MPT. In other words, the MPT is generated by changes in orbital geometry.

\section{Acknowledgments, Samples, and Data}

Data and Code Availability: The data and computer code used in this analysis are available on OpenBU, which is FAIR-compliant, and can be accessed through a globally unique and eternally persistent identifier, https://hdl.handle.net/2144/40340 P. This dataset is distributed under the terms of the Creative Commons Attribution-ShareAlike 4.0 License (http://creativecommons.org/licenses/by-sa/4.0).

Team list: The team includes Robert Kaufmann (RK) and Felix Pretis (FP).

Author contributions: This project was conceived by RK and FP. RK compiled the data from the statistical model and FP did the statistical analysis to identify impulses and steps. RK and FP write the manuscript, designed the tables, and created the figures together.

Competing Interests: The authors have no financial or non-financial interests associated with the material in this manuscript. 
Acknowledgements: We thank David F Hendry, Luke Jackson, and Katarina Juselius for helpful comments and suggestions. Financial support from the Robertson Foundation and British Academy is gratefully acknowledged.

\section{References}

Ashwin, P. amd P. Ditevsen, 2015, The mideele Pleistocene transition as a generic bifurcation on a slow manifold, Climate Dynamics, 45:2683-2695.

Castle, J.L., Doornik, J.A., Hendry, D.F., \& Pretis, F., (2015). Detecting location shifts during model selection using step-indicator saturation. Econometrics, 3, 240-264.

Crucifix, M., (2013). Why could ice ages be unpredictable? Climate of the Past, 9:2253-2267.

Davidson, J. E., Stephenson, D. B., \& Turasie, A. A., (2016). Time series modeling of Paleoclimate data. Environmetrics, 27(1), 55-65.

Ditlevsen, P., Mitsui, T. and Crucifix, M., (2020), Crossover and peaks in the Pleitocene climate spectrum:understanding frm simple ice age models, Climate Dynamics, 54:1801-1818.

Engle, R.F., \& Granger, C.W.J., (1987). Cointegration and error correction: representation, estimation, and testing, Econometrica, 55(2), 251-276.

EPICA Community Members, (2004). Eight glacial cycles from an Antarctic ice core, Nature, $429,623-628$.

Elderfield, H., Ferretti P., Crowhurst, S., McCave, I.N., Hodell, D., \& Piotrowski, A.M., (2012). Evolution of ocean temperature and ice volumne through the mid-Pleisocene climate transition, Science 337(6096):704-709..

Ericsson, N.R., (2017), How Biased Are U.S. Government Forecasts of the Federal Debt? International Finance Discussion Papers 1189.

Ganopolski, A., \& Brovkin, V., (2017). Simulation of climate, ice sheets, and $\mathrm{CO}_{2}$ evolution during the last four glacial cycles with an earth system model of intermediate complexity, Climate of the Past, 13, 1695-1716.

Ganopolski, A., Winkelmann, R., \& Schellnhuber, H.J., (2016). Critical insolation- $\mathrm{CO}_{2}$ relation for diagnosing past and future glacial inception, Nature 529: 7585: 200-U159.

Goldewijk, K.K., Beusen, A., Doelman, J. \& Stehfest, E., (2017), Anthropgenic land use estimates for the Holocene - HYDE 3.2, Earth System Science Data, 9(2):927-953. 
Harbo, I., Johansen, S., Nielsen, B., \& Rahbek, A., (1998). Asymptotic inference on cointegrating rank in partial systems. Journal of Business \& Economic Statistics, 16(4), 388-399.

Hendry, D., (1994). Dynamic Econometrics, Oxford: Oxford University Press..

Hendry, D. F., \& Richard, J. F. (1982). On the formulation of empirical models in dynamic econometrics. Journal of Econometrics, 20(1), 3-33.

Hoenisch, B., et al, (2009). Carbon dioxide concentrations across the Mid-Pleistocene Transition, Science 324:1551-1554, 2009.

Imbrie, J., et al., (1993). On the structure and origin of major glaciation cycles. Part 2. The 100,000-year cycle, Paleoceanography 8:699-735, 1993.

Jansen, J.H.F., Kuijpers, A., \& Troelstra, S.R., (1986). A Mid-Brunhes climatic event: long-term changes in global atmosphere and ocean circulation Science 750:619-622.

Johansen, S., (1992). Cointegration in partial systems and the efficiency of single-equation analysis. Journal of Econometrics, 52(3), 389-402.

Jouzel, J., et al.,. (2007). Orbital and millennial Antarctic climate variability in Antarctic climate variability over the past 800,000 years, Science, 317, 793-796.

Juselius, K., (2006). The cointegrated VAR model: methodology and applications. Oxford, Oxford University.

Kaufmann, R.K., \& Juselius, K., (2013). Testing hypotheses about glacial cycles against the observational record, Paleoceanography 28, 1-10, doi:10.1002/palo.20021.

Kaufmann, RK \& Juselius, K., (2016), Testing Competing Forms of the Milankovitch Hypothesis: A Multivariate Approach, Paleoceanography. 31, doi:10.1002/2014PA002767.

Kaufmann, RK \& Pretis, F., (2021). Understanding glacial cycles: a multivariate disequilibrium approach, Quaternary Science Review, 106694.

Lisiecki, L. E., \& Raymo, M. E., (2005), A Pliocene-Pleistocene stack of 57 globally distributed benthic D18O records, Paleoceanography, 20, 1-17.

Loulergue, L. A. Schilt, R. Spahni, V. Masson-Delmotte, T. Blunier, B. Lemieux, J. Banola, D. Ranaud, T.F. Stocker, J. Chappellaz, 2008, Orbital and millennial-scale features of atmospheric CH4 over the past 800,00 years, Nature 453:383-386.

Luthi, D., et al., (2008), High-resolution carbon dioxide concentration record 650,000-800,000 years before present, Nature, 453, 379-382, 2008

Maasch, K., , and B. Saltzman, 1990: A low-order dynamical model of global climatic variability 
over the full Pleistocene. J. Geophys. Res., 95 , 1955-1963.

Martinez-Garcia, A., Rosell-Mele, A., Geibert, W., Gersonde, R., Masque, P., Gaspari, V., and Barbante, C. (2009), Links between iron supply, marine productivity, sea surface temperature, and CO2 over the last .1. Ma, Paleoceanography, 24, 1-14.

Miller, J.I., (2019). Testing cointegrating relationships using irregular and non-contemporaneous series with an application to paleoclimate data, Journal of Time Series Analysis, DOI: $10.1111 /$ jtsa.12469.

Paillard, D. (1998), The timing of Pleistocene glaciations from a simple multiple-state climate model, Nature, 391, 378-381.

Paillard, D., (2001), Glacial Cycles: Toward a New Paradigm, Review of Geophysics, 39, 3: 325346.

Paillard, D., Labeyrie, L., \& Yiou, P. (1996). Macintosh program performs time-series analysis, Eos Trans. $A G U, 77,379$.

Paillard, D., and F. Parrenin, 2004, The Antarctic ice sheet and the triggering of deglaciations, Earth and Planetary Science Letters, 227:263-271.

Parrenin, F. and D. Paillard, 2012, Terminations VI and VIII( 530 ad $720 \mathrm{kyr}$ BP) tell us the importance of obliquity and precession in triggering of deglaciations, Climate of the Past, 8:2031-2037.

Parrenin, F., et al., (2007). The EDC3 chronology for the EPICA Dome C ice core, Clim. Past, 3, 485-497, https://doi.org/10.5194/cp-3-485-2007.

Past interglacials Working Group of Pages, (2016). Interglacials of the last 800,00 years, Rev. Geophys., 54,162-219.

Pretis, F., Mann, M.L., \& Kaufmann, R.K. (2015), Testing competing models of the temperature hiatus: assessing the effects of conditioning variables and temperature uncertainties through sample-wide break detection, Climatic Change, 131(4): 705-718.

Pretis, F., Reade, J., \& Sucarrat, G., (2018). Automated General-to-Specific (GETS) regression modeling and indicator saturation methods for the detection of outliers and structural breaks. Journal of Statistical Software, 86(3).

Pretis, F., Schneider, L., Smerdon, J. E., \& Hendry, D. F., (2016). Detecting Volcanic Eruptions in Temperature Reconstructions by Designed Break-Indicator Saturation. Journal of Economic Surveys, 30(3), 403-429.

Roe, G.H.: (2006). In defense of Milankovitch, Geophysical Research Letters, 33(L23703). 
Roe, GH. Ad R.S. Lindzen, (2001), A one-dimensional model for the interacton between continental scale ice sheets and atmospheric stationary waves, Climate Dynamics, 17:479-487.

Ruddiman, W.F., and Raymo, M.E.: (2003), Orbital insolation, ice volume, and greenhouse gases, Quaternary Science Reviews, 22:1597-1629.

Ruddiman, W.F., (2005). The early anthropogenic hypothesis: a year later, Climatic Change, 69: 427-434.

Ruddiman, W.F., (2007). The early anthropogenic hypothesis: challenges and responses, Rev. Geophys. 45:1-37.

Ruddiman, W.F. and Raymo, M.E. (2003): A methane-based time scale for Vostok ice, Quaternary Science Reviews 22(2-4): 141-155.

Schneider, L., Smerdon, J.E., Pretis, F., Hartl-Meier, C., \& Esper, J., (2017). A new archive of large volcanic events over the past millennium derived from reconstructed summer temperatures. Environmental Research Letters, 12(9), 094005.

Siddall, M. et al., (2003). Sea-Level fluctuations during the last glacial cycle, Nature, 423: 853858.

Stephens, L., et al., (2019). Archaeological assessment reveals Earth's early transformation through land use, Science 365(6456):897-902.

Tzedakis, P.C., Crucifix, M., Mitsui, T., \& Wolff, E.M., (2017). A simple rule to determine which insolation cycles lead to interglacials, Nature 542:427-432.

Tzedakis, P.C., Raynaud, D., McManus, J.F., Berger, A., Brovkin, V., \& Kiefer, T., (2009). Interglacial diversity, Nature Geoscience, 12:751-755.

Tzedakis, P.C. Wolff, E.W. Skinner, L.C. Brovkin, V. Hodell, D.A. MaManus, J.F. \& Raynard, D., (2012). Can we predict the duration of an interglacial, Clim. Past 8:1473-1485.

Tziperman, E., Raymo, M.E., Huybers, P., \& Wunsch, C., (2006). Consequences of pacing the Pleistocene $100 \mathrm{Kyr}$ ice ages by nonlinear phase locking to Milankovitch forcing, Paleoceanography, 21:PA4206 doi:10.1029/2005PA001241.

Wolff, E.W. et al., (2006). Southern ocean sea-ice extent, productivity and iron flux over the past eight glacial cycles, Nature, 440: 491-496.

Wolff, E.W. Rankin, A.M., \& Rothlisberger, R., (2003). An ice core indicator of Antactic sea ice production? Geophys. Res. Lett. 30 doi:10.1029/2003GLO18454. 
607 Wunsch, C. (2004), Quantitative estimate of the Milankovitch-forced contribution to observed 608 Quarternary climate change, Quaternary Science Reviews 23:1001-1012

609 Yin, Q., (2013)., Insolation-inducd mid-Brunhes transition in Southern Ocean ventilation and 610 deep-ocean temperature, Nature 494:222-225.

611 Yin, Q.Z., \& Berger, A., (2012). Individual contribution of insolation and $\mathrm{CO}_{2}$ to the interglacial 612 climates of the past 800,000 years, Climate Dynamics 38:709-724.

613 Yule, G. (1929). An Introduction to the Theory of Statistics, C. Griffin and Co., London. 614 


\section{Figure Captions}

Figure 1(a) Experimental data for the time series that responds quickly $(T)$ that is generated by the van der Pol Oscillator is given by the black line; the blue line represents values simulated by the CVAR model. Figure 1(b) Experimental data for the time series that responds slowly $(I)$ that is generated by the van der Pol Oscillator is given by the black line; the blue line represents values simulated by the CVAR model.

Figure 2 The observed values for temperature (black line) and values simulated by the system model conditioned only on the four variables for solar insolation (red line). Thick portions of the red line represent time steps in which the simulation error is significantly different from zero (non-zero error). Red circles represent time steps when the simulation error is an (innovational) outlier. The light gray area is the out-of-sample forecast period; MIS 11 is shaded dark gray. (b) same as above for carbon dioxide, (c) same as above for methane, (d) same as above for land ice, (e) same as above for $\mathrm{Na}$, (f) same as above for $\mathrm{SO}_{4}$, (g) same as above for sea level, (h) same as above for $S S T^{2}$.

Figure 3 The number of outliers (red spikes) and non-zero errors (darkly shaded) for each time step. Marine isotope stages are indicated by alternating areas of shading.

\footnotetext{
${ }^{2}$ Note that the series of SST exhibits non-zero simulation errors nearly throughout the sample, suggesting a nonzero bias throughout the observational record - simulated model values persistently exceed observations.
} 

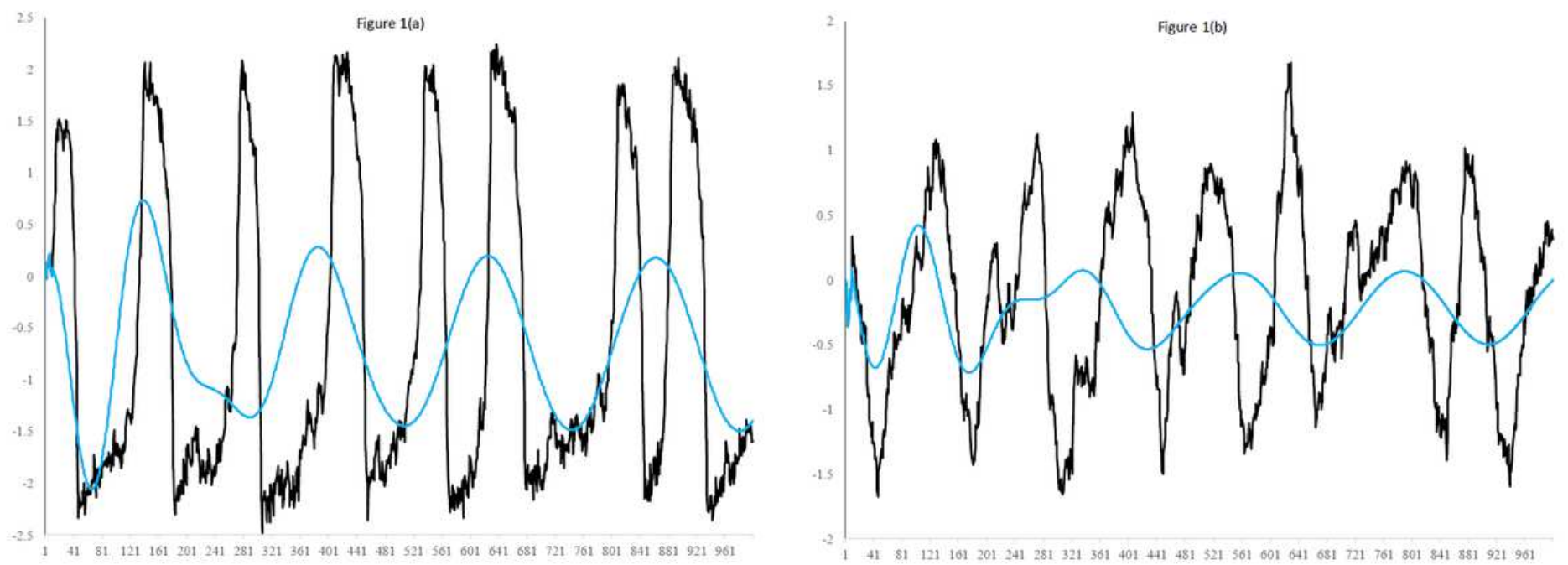

Figure 1

1(a) Experimental data for the time series that responds quickly $(T)$ that is generated by the van der Pol Oscillator is given by the black line; the blue line represents values simulated by the CVAR model. Figure 1(b) Experimental data for the time series that responds slowly (I) that is generated by the van der Pol Oscillator is given by the black line; the blue line represents values simulated by the CVAR model.
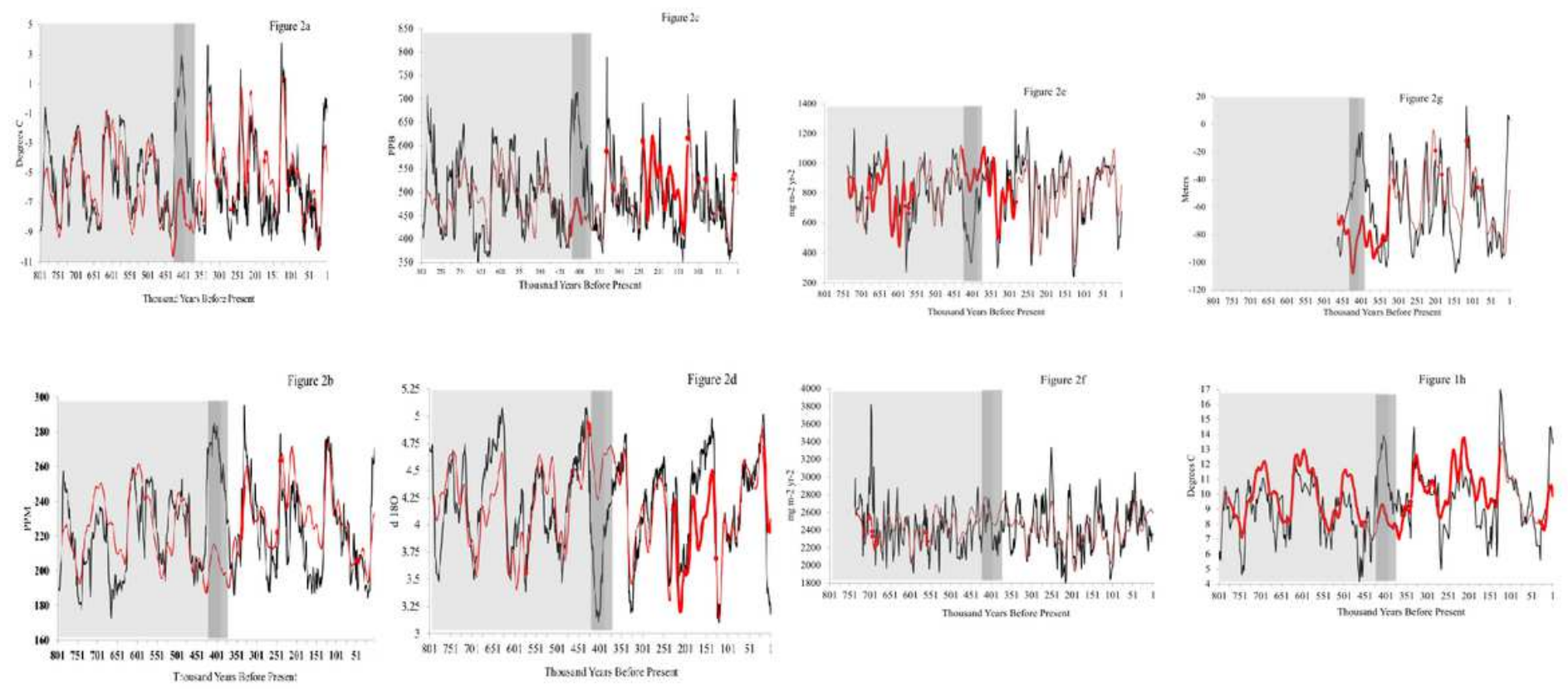

\section{Figure 2}


The observed values for temperature (black line) and values simulated by the system model conditioned only on the four variables for solar insolation (red line). Thick portions of the red line represent time steps in which the simulation error is significantly different from zero (non-zero error). Red circles represent time steps when the simulation error is an (innovational) outlier. The light gray area is the out-of-sample forecast period; MIS 11 is shaded dark gray. (b) same as above for carbon dioxide, (c) same as above for methane, (d) same as above for land ice, (e) same as above for $\mathrm{Na}$, (f) same as above for $\mathrm{SO} 4$, (g) same as above for sea level, (h) same as above for SST2.

Figure 3

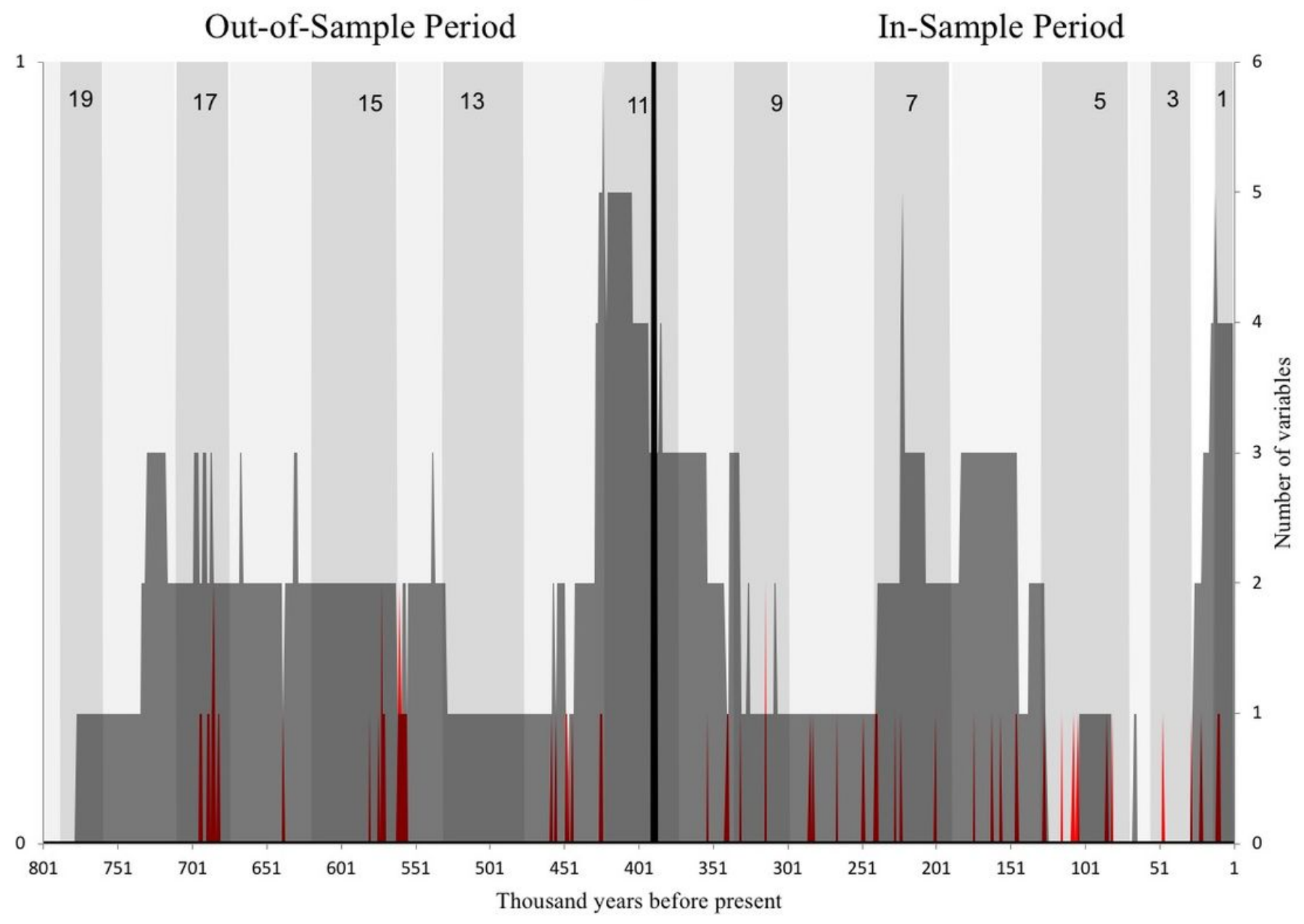

\section{Figure 3}

The number of outliers (red spikes) and non-zero errors (darkly shaded) for each time step. Marine isotope stages are indicated by alternating areas of shading.

\section{Supplementary Files}

This is a list of supplementary files associated with this preprint. Click to download. 
- KaufmannPretisSupporting.pdf

- Table1.pdf

- Table2.pdf

- Table3.pdf 